Gdańsk 2019, Nr. 41

https://doi.org/10.26881/sgg.2019.41.02

Anna Averina

Staatliche Landesuniversität Moskau

https://orcid.org/0000-0002-3117-6362

\title{
Epistemizitätsmarker aus der Sicht ihrer Egozentrizität \\ (eine kontrastive Analyse von Epistemizitätsmarkern im Deutschen und im Russischen)
}

\begin{abstract}
Im vorliegenden Beitrag werden Epistemizitätsmarker des Deutschen und des Russischen nach dem Grade ihrer Egozentrizität eingeteilt. Die erste Gruppe schließt solche Egozentrika ein, die im Redemodus sowie in illokutiv selbständigen Sätzen verwendet werden können. Zur zweiten Gruppe gehören Epistemizitätsmarker, die sowohl in illokutiv selbständigen Nebensätzen als auch im narrativen Textmodus möglich sind. Zur dritten Gruppe kann man solche Marker der Epistemizität zählen, die in allen Interpretationsmodi auftreten und in illokutiv unselbständigen Nebensätzen gebraucht werden können.
\end{abstract}

Schlüsselwörter: Epistemizitätsmarker, Egozentrizität, Deiktizität, Interpretationsmodi, illokutiv unselbständige Nebensätze

Epistemic markers from the perspective of their egocentricity (contrastive analyses of epistemic markers in the German and Russian languages). - The article deals with the epistemic markers in German and in Russian which can be divided into three groups according to their egocentric degree. The first group includes epistemic markers beeing able to be used in the speech mode and in peripheral clauses as well. The epistemic markers which can be used in peripheral clauses and in narrative text belong to the second group. The third group is built by epistemic markers which can be used in central and peripheral clauses, in narrative text and in the direct speech as well.

Keywords: Epistemic markers, egocentricity, deicticity, modes of interpretation, peripheral clauses

\section{Zielsetzung und Fragestellung}

In meinem Beitrag1 möchte ich auf die Frage eingehen, wie Epistemizitätsmarker im Deutschen und im Russischen nach dem Grad ihrer Egozentrizität, d.h. nach der Entfernung vom Sprecher differenziert werden können. In diesem Zusammenhang möchte ich Antworten auf folgende Fragen geben:

1 Ich bedanke mich bei Herrn Professor Werner Abraham für seine wertvollen Hinweise auf der Konferenz Modalität / Aspektualität und Temporalität aus kontrastiver und typologischer Sicht im September 2018 in Gdańsk. 
(1) Wie korrelieren die Begriffe „Egozentrizität" und „Deixis“ miteinander?

(2) Welche Kontexte dienen als Test für die Bestimmung der Egozentrizität?

(3) Wie können Epistemizitätsmarker nach dem Grade ihrer Egozentrizität differenziert werden?

(4) Welche Parallelen können nach dem Grade der Egozentrizität bei epistemischen Markern des Deutschen und des Russischen gezogen werden?

Meine Untersuchung basiert auf der Analyse des deutschen Korpus DECOW 16A. Um das Funktionieren einzelner Phänomene in einem größeren Kontext und in unterschiedlichen Textsorten zu veranschaulichen, habe ich Belege aus den Novellen von Stefan Zweig, aus dem Roman von Patrick Süskind „Das Parfum“ sowie aus einer wissenschaftlichen Ausgabe benutzt. Russische Belege sind dem russischen Nationalkorpus entnommen.

\section{Die Korrelation der Begriffe Epistemizität und Deiktizität, Modalität und Deiktizität}

Es gibt keine Eindeutigkeit in Bezug auf die Frage, in welchem Zusammenhang solche Begriffe wie Epistemizität und Deiktizität zueinander stehen.

Deiktika werden als egozentrisch von Diewald (1991) und von Apresjan (1995) betrachtet. Diewald behandelt Deixis als Denotation von Gegenständen aus der egozentrischen Perspektive, vgl.:

„Egozentrismus ist also die Grundlage des deiktischen Prozesses, da dieser seine notwendige Verankerung in der Origo (dem Ego des Sprechers) hat [...]“ (Diewald 1991: 38).

Sie unterscheidet zwischen den starken (lexikalischen) und den schwachen (grammatischen) Deiktika: Lexikalische deiktische Zeichen haben ein Deixisobjekt im realen Zeigfeld bzw. in einem abgeleiteten Zeigfeld, z.B.: A: Wann wart Ihr in der Ausstellung? - B: Gestern. Grammatische Zeichen haben kein Deixisobjekt im eigentlichen Sinne. Sie verweisen stattdessen auf ein anderes sprachliches Zeichen, das sie modifizieren, vgl.: Sie besuchten die Ausstellung, wo durch das deiktische Zeichen kein autonomes Deixisobjekt lokalisiert wird, stattdessen modifiziert das Tempusgrammem die sprachlich enkodierte Szene in temporaldeiktischer Sicht (nach Diewald 2013: 83-84). Die schwachdeiktische Relation geht von der Interpretation durch den Rezipienten aus (ebd.: 85).

DiEWALD (1991) spricht von der objektalen, personalen, temporalen, lokalen, modalen und der evidentiellen (2013) deiktischen Dimension. ${ }^{2}$ Bei der Entfernungsbeimessung wird die Entfernung zwischen der Origo und dem Ziel des Hinweisens bewertet (Diewald 2013: 85). Auf die Korrelation der Deiktizität und Egozentrizität weist auch Apresjan (1995) hin.

2 Laut Diewald geben die Dimensionen der Deixis Auskunft über die Art des Deixisobjekts. So umfasst z.B. die lokale Dimension alle Deiktika, deren Deixisobjekt ein Ort ist (Diewald 1991: 152). Die temporale Dimension der Deixis fixiert einen Zeitbereich durch Rückbezug auf die mit der realen Sprechzeit gegebene Origo (ebd.: 268). Die personale Dimension betrifft Kontextelemente, die der Kategorie Person zugehören (ebd.: 202). Die objektale Dimension hat sowohl mit der personalen als auch mit der lokalen Dimension gewisse Ähnlichkeiten, „ohne dass sie mit einer von ihnen identifiziert werden“ (ebd.: 227). Im Falle der modalen Dimension bewertet der Sprecher einen Sachverhalt als faktisch oder als nicht faktisch (ebd.: 239). 
Für die Bewertung der Egozentrizität wird die Entfernung von der Origo berücksichtigt. Als semantische Grundlage der deiktischen Lexik dienen die Begriffe „Ich“, „ego“ und „Sprecher“. Apresjan unterscheidet zwischen der primären und der sekundären Deixis. Die primäre Deixis ist die Dialogdeixis bzw. Rededeixis, die für einen Kommunikationsprozess typisch ist: Der Sprecher und der Hörer sehen einander. Die sekundäre Deixis, oder die narrative Deixis ist mit der Redesituation nicht verbunden. Es handelt sich um die Deixis der Nacherzählung bzw. um die narrative Deixis. Deiktische Wörter dienen zum Ausdruck des Fremdbewusstseins und haben entweder eine kataphorische oder eine anaphorische Funktion (vgl. APRESJAN 1995: 276).

Einen anderen Standpunkt vertritt PAdučEva (2010). Sie betrachtet Deiktizität und Egozentrizität als unterschiedliche Entitäten. Deiktisch sind Einheiten, die ein Objekt hinsichtlich des Ortes, der Zeit sowie der Situation durch seinen Bezug zum Sprechakt, seinen Teilnehmern sowie zu einem bestimmten Kontext identifizieren. Sie zählt nicht zu deiktischen Kategorien solche wie Modus, Modalität sowie Marker der illokutiven Funktion (z.B. Assertion) (Padučeva 2010: 245). Sie bezeichnet diese Phänomene als Egozentrika, da ihre Semantik einen Sprecher voraussetzt. So enthält z.B. der Satz Bylo by sejčas letoß’ / Wenn es jetzt doch Sommer wäre! keine deiktische Komponente (Der Sprecher will...). Als kennzeichnendes Merkmal der Deixis, das sie von anderen Egozentrika unterscheidet, ist ihre Identifizierungsfunktion (Identifizierung von Objekten, Zeit- und Raumrelationen usw.) (vgl. ebd.: 246).

Deiktizität und Egozentrizität können nach Padučeva Schnittstellen aufweisen, sie sind aber nicht identisch. Marker der Egozentrizität setzen einen Sprecher voraus, während eine deiktische Projektion einen Sprecher nicht immer impliziert. So wird z.B. im Satz:

(1) Zdes'ne kurjat ,Hier wird nicht geraucht

das Wort hier zum Marker des Ortes, in dem sich der Adressat befindet, setzt aber den Sprecher nicht voraus (ebd.: 261). Vergleichen wir auch einen Beleg aus Fillmore (1981):

(2) I may be in room 2114 ,

wo die Zeitform nicht die Zeit impliziert, wann der Zettel geschrieben wurde, sondern die Zeit, wann er gelesen wird (ebd.).

Zu den egozentrischen Einheiten zählt Padučeva solche Wörter wie z.B. kažetsja (ein Einführungswort mit der Semantik „es scheint mir zu sein“), Wörter mit bewertender Semantik usw.

M.E. ist Epistemizität deiktisch - die Darstellung der Nichtfaktizität der Situation tritt als Objekt auf, auf das die Origo hinweist. Wenn die epistemische Struktur nur für solche Situationen typisch ist, wenn der Sprecher als deiktisches Zentrum auftritt, sind sie egozentrisch. Weniger egozentrisch sind Epistemizitätsmarker, die die Einstellung nicht nur der 1. Person, sondern auch der 3. Person kodieren können. Des Weiteren werde ich von Entfernungsstufen der Egozentrika sprechen, in deren Rolle Epistemizitätsmarker auftreten - darunter wird

3 Bei der Transliteration habe ich den DIN Standard (DIN 1460) benutzt. DIN 1460: 1982 ist die deutsche Adaption von ISO/ R 9: 1968. 
ihre Entfernung von der Origo gemeint. Als Test für eine solche Differenzierung dienen unterschiedliche Interpretationsmodi.

\section{Die Modi des Zeigens als Grundlage für die Interpretation der Egozentrika}

Die Frage in Bezug auf unterschiedliche Modi des Zeigens wurde schon von Bühler erhoben:

„Die Modi des Zeigens sind verschieden; ich kann ad aculos demonstrieren und in der situationsfernen Rede dieselben Zeigwörter anaphorisch gebrauchen. Es gibt noch einen dritten Modus, den wir als Deixis am Phantasma charakterisieren werden" (BÜHLER 1978: 80).

Diewald unterscheidet zwischen Realdeixis, Deixis am Phantasma (Versetzungsdeixis) und Textdeixis. Eine solche Auseinanderhaltung hält Diewald für die Textsortendifferenzierung relevant (Diewald 1991: 125-126). Laut Diewald ist die Realdeixis der grundlegende Zeigmodus, da der außersprachliche Kontext der Äußerung als Zeigfeld auftritt. Die Deixis am Phantasma ,geht von einer imaginierten bzw. verschobenen oder versetzten Origo aus, die an einer anderen Stelle als die reale Origo des Sprechers lokalisiert ist" (ebd.: 126). Als Deixisobjekt der Textdeixis tritt ein Textsegment auf (vgl. ebd.).

Bei der Auseinanderhaltung von Deiktika spricht Apresjan (1995) von der Redesituation und Narration, es handelt sich also um den narrativen Modus und um den Textmodus. Auf unterschiedliche Interpretationsmodi von Egozentrika weist PADUČEva (2010) hin. Sie spricht von primären und sekundären Egozentrika. Für die Interpretation von egozentrischen Elementen benutzt Padučeva mehrere Interpretationsmodi:

- einen Dialogmodus, oder Redemodus, wenn die Sprecherrolle von einem realen Sprecher erfüllt wird und

- einen narrativen Modus, wenn in der Funktion des Sprechers entweder eine der Figuren oder der Erzähler auftritt.

Nach Padučeva können nicht primäre Egozentrika in einem narrativen Modus verwendet werden.

In Bezug auf den narrativen Modus möchte ich sagen, dass er nicht einheitlich ist. Man kann eine weitere Differenzierung machen - der narrative Modus kann in zwei Gruppen unterteilt werden, und zwar:

- in den narrativen Textmodus, wenn der Erzähler auktorial ist und die Handlung aus der Außenperspektive dargestellt wird, und

- in den narrativen Textmodus, wenn die Handlung aus der Innenperspektive dargestellt wird und in der Sprecherrolle der Ich-Erzähler auftritt.

Padučeva spricht noch von dem syntaktischen Modus, wenn ein egozentrisches Element in einem Nebensatz verwendet wird, vgl.:

(3a) Zina sčitaet, čto Ivan, bezuslovno, vernëtsja (PADUČEVA 2010: 266).

,Sina ist der Ansicht, dass Ivan natürlich zurückkommt'. 
Sekundäre Egozentrika können nach Padučeva untergeordnet werden, d.h. in Nebensätzen verwendet werden, während das für primäre nicht der Fall ist. M.E. sollte hier aber berücksichtigt werden, dass es illokutiv selbständige und illokutiv unselbständige Sätze gibt - die Einbettung von Egozentrika in illokutiv unselbständige Nebensätze kann ungrammatisch sein, vgl.:

$\left(3 b^{*}\right)$ Zina sožaleet, čto Ivan, bezuslovno, vernëtsja. ,Sina bedauert, dass Ivan unbedingt zurückkommt

Durch die Analyse der Verwendung von Epistemizitätsmarkern in unterschiedlichen Interpretationsmodi kann man sie nach ihrer Entfernung von dem Sprecher differenzieren. Als Test für eine solche Differenzierung dienen also folgende Modi:

(1) Redemodus, oder Realmodus, in dem die Sprechereinstellung kodiert wird.

(2) Textmodus mit dem Ich-Erzähler in der Sprecherrolle.

(3) Textmodus mit dem auktorialen Sprecher.

(4) syntaktischer Modus. Der syntaktische Modus ist nicht einheitlich: Es gibt illokutiv selbständige und illokutiv unselbständige Nebensätze. Nach ihrer Verwendung in Nebensätzen sind Epistemizitätsmarker uneinheitlich.

\section{Epistemizitätsmarker im Deutschen und im Russischen aus der Sicht ihrer Entfernung von dem Sprecher}

\subsection{Typen von Epistemizitätsmarkern nach ihrer Entfernung vom Sprecher}

Nach ihrer Entfernung vom Sprecher möchte ich Epistemizitätsmarker in drei Gruppen einteilen: in die Gruppe der nahen, der medialen und der entfernten Egozentrika.

- Nahe oder primäre Egozentrika geben die Einstellung der Ich-Person wieder. Sie sind nur im Dialogmodus möglich, wenn die Handlung aus der Innenperspektive, d.h. aus der Perspektive eines realen Sprechers dargestellt wird. Sie können auch in illokutiv selbständigen Nebensätzen verwendet werden.

- Mediale Egozentrika können in illokutiv selbständigen Nebensätzen sowie in einem narrativen Text mit einem auktorialen Sprecher verwendet werden. Ihre Verwendung in illokutiv unselbständigen Nebensätzen ist nicht möglich.

- Entfernte Egozentrika als lexikalische Epistemika (LeIss 2011) treten in allen Interpretationsmodi ohne Einschränkungen auf und können somit die Einstellung der zitierten Origo wiedergeben. Sie können in jedem Nebensatztyp verwendet werden.

\subsection{Nahe Egozentrika}

Zu nahen oder primären Egozentrika zähle ich den Verumfokus sowie betonte Partikeln im Deutschen und das verifikative Rhema im Russischen. Nach Höhle geht es beim Verumfokus um einen Fokusakzent im Deutschen, der die Wahrheit der Proposition kodiert 
(vgl. HöHLE 1988, 1992). Dabei wird in der linken Satzperipherie ein Pitch-Akzent realisiert, dessen Funktion ist es, die notwendige Fokussierung zu kodieren, vgl.:

(4) Karl HAT den Hund getreten (HöHLE 1988: 3-4).

Im Russischen wird der Terminus das verifikative Rhema verwendet (vgl. Janko 2008).

Verumfokus und betonte Partikeln sind für den Redemodus typisch und haben eine Adressatenanbindung. Ihre Verwendung in einem narrativen Textmodus mit dem Ich-Erzähler in der Sprecherrolle ist auch möglich.

\subsubsection{Verumfokus im Deutschen}

Das Verumphänomen verweist auf die Faktizität des Sachverhalts und wird von manchen Sprachforschern als Kontrastfokus behandelt (STOMMEL 2012). Laut BLÜHDORN (2012) können Sätze mit Verum als epistemisch charakterisiert werden. So ist z.B. der Satz

(5) \{Martin glaubt nicht, dass Otto nach dem Konzert müde war, aber...\} IANnas meinung nach WAR $\mid$ otto nach dem konzert müde

$\rightarrow$ Annas Meinung nach ist es wahr, dass es nach dem Konzert der Fall war, dass Otto müde war.

epistemisch, da der Proposition ein Wahrheitswert zugeordnet wird (BLÜHDORN 2012: $142-143)$.

Verumfokus weist Affinitäten zu Modalpartikeln auf, weil er in illokutiv unselbständigen Nebensätzen nicht fungieren kann, worauf auch Abraham hingewiesen hat: „Not only VF, but also MP has a truth-valuating function“" (ABRAHAm 2017: 174). Das kann am folgenden Beleg beobachtet werden:

(6a) aber Hanna meint, er HAT gelogen (HöHLE 1992: 123).

In (6a) wird die Einstellung der 3. Person kodiert, die auch der Sprecher teilt. Verumfokus ist für den Redemodus typisch: Der Sprecher will im Kommunikationsprozess zeigen, dass seine Einstellung wahr ist. Die Kodierung des Verums in einem illokutiv unselbständigen Nebensatz ist nicht möglich - in diesem Zusammenhang weist das Verumphänomen Affinitäten mit Modalpartikeln auf, vgl.:

(6b) *aber Hanna bedauert, dass er gelogen HAT.

\subsubsection{Betonte Partikeln JA und DOCH}

Betonte Partikeln unterscheiden sich wesentlich von unbetonten Modalpartikeln dadurch, dass die letzteren logische Relationen kodieren, während betonte Partikeln einen Kontrast schaffen (über den modalen Kontrast vgl. Meibau er 1994). Nicht alle Sprachforscher teilen die Meinung, dass betonte Partikeln Verumfokus kodieren. Laut Meibauer (1994) wird in Sätzen mit betonten Partikeln ein modaler Kontrast wiedergegeben, EGG und ZimmerMANN (2012) sind der Ansicht, dass betontes DOCH Verum kodiert, auch BLÜHDORN 
(2012) weist darauf hin, dass es Affinitäten zwischen der Kodierung des Verums durch betonte Partikeln und durch die Betonung des Subjunktors gibt, indem er folgende Belege anführt:

(7) $\{$ A-Die Abgeordneten scheinen nicht rechtzeitig angereist zu sein\}

a. $B$ - die abgeordnneten sind $J A \mid$ rechtzeitig angereist $\{/$ was sind denn $D A S$ für geRÜCH $\mid$ te $\}$

b. $B$ - die abgeordneten sind $D O C H \mid$ rechtzeitig angereist $\{/ /$ die/AUF $\mid$ regung war um $\mid S O N S T\}$

(BLÜHDORN 2012: 160).

Ich finde den Fall (7a) problematisch, weil betontes JA einen komplementären Kontrast kodiert: Durch betontes JA wird gezeigt, dass eine Alternative aus einer Alternativenmenge ausgewählt und hervorgehoben wird, während im Falle mit dem betonten DOCH, das einen kontradiktorischen Kontrast zum Ausdruck bringt, eine Alternative einer anderen gegenübergestellt wird. Diese Partikel ist für den Redemodus charakteristisch, wie das der Beleg (7b) deutlich veranschaulicht, sie kann auch in einem narrativen Text mit dem Ich-Erzähler verwendet werden, vgl.:

(8a) Sie weiß das und lässt seine Erklärungsversuche regungslos über sich ergehen. Und DOCH ist ihr Sorge ins Gesicht geschrieben: ob der Gefährte es gut überstehen wird, wenn er es erfährt? (DECOW 16A).

Ebenso wie Verumfokus kann betontes DOCH in illokutiv selbständige Nebensätze eingebettet werden, vgl. (8b), die Einbettung dieser Partikel in illokutiv unselbständige Sätze ist nicht akzeptabel, vgl. (8c):

(8b) A: Ibr ist keine Sorge ins Gesicht geschrieben.

B: Aber ich sage, dass ihr DOCH Sorge ins Gesicht geschrieben ist.

$\left(8 \mathrm{c}^{*}\right)$ Aber ich bedauere, dass ihr DOCH Sorge ins Gesicht geschrieben ist.

\subsubsection{Das verifikative Rhema im Russischen}

Eines der Mittel, das auf die Faktizität des Sachverhalts durch die Negation der vorangehenden Aussage hinweist, ist die Betonung des Prädikats bzw. Prädikativs, so wie man das am folgenden Beispiel beobachten kann (durch $\$ wird der Tonfall markiert), vgl.:

(9) S 1: Uvažaemyj Ivan Ivanovič, ja Vam ne protivoreču.

S 2: Protivo||rečite.

,S 1: Sehr geehrter Ivan Ivanovitsch, ich widerspreche Ihnen nicht.

S 2: DOCH!

Einen solchen Satztyp bezeichnet Adamec als verifikativ: Der Sprecher hat nicht erwartet, dass der Gesprächspartner seine Einstellung der des Gesprächspartners gegenüberstellt. Es handelt sich nicht um die neue Information, sondern um die Reaktion des Sprechers auf die Meinung des Gesprächspartners, um die Korrektur solcher Meinung. Als Kern von verifikativen Aussagen tritt ein Verb auf, genauer gesagt, seine positive oder negative Form (ADAMEC 1966: 27-28). Diese Idee wurde von Janko weiterentwickelt: Sie hat den Begriff das verifikative Rhema eingeführt. Das verifikative Rhema entsteht oft in der Polemik, dabei enthält ein Satz Negation (JAN Ko 2008: 156). 
Das verifikative Rhema kann nur in einen illokutiv selbständigen Satz eingebettet werden, vgl.:

(10) A: Ja emu ne protivoreču.

$B:$ A ja govorju, čto protivo| |recite.

,A: Ich widerspreche ihm nicht.

$B:$ Aber ich meine, dass Sie widerSPRECHen'

( ${ }^{*}$ A ja sožaleju, čto protivo||rečite).

*Aber ich bedauere, dass Sie widerSPRECHen

Es ergibt sich, dass zu primären Egozentrika, die in illokutiv selbständigen Nebensätzen sowie im Redemodus möglich sind, intonatorisch markierte Mittel des Deutschen und des Russischen gehören.

\subsection{Mediale Egozentrika}

Zu medialen Egozentrika zähle ich Modalpartikeln im Deutschen und im Russischen, Modalverben in sekundärer Bedeutung im Deutschen und Modaladverbien im Russischen. Ihre Eigenschaft besteht darin, dass sie in illokutiv selbständigen Nebensätzen und in einem narrativen Textmodus verwendet werden können, indem sie die Einstellung des Sprechers immer kodieren.

\subsubsection{Modalpartikeln im Deutschen}

Modalpartikeln können in allen Textmodi verwendet werden - im Redemodus, in einem narrativen Text mit einem auktorialen Sprecher in der Funktion des Erzählers sowie in einem narrativen Text mit dem Ich-Erzähler. Eine der Funktionen der Modalpartikeln besteht darin, logische Relationen auf der satzübergreifenden Ebene zu schaffen. Nicht umsonst werden sie häufig in wissenschaftlichen Texten verwendet, um zu überzeugen und zu argumentieren, vgl.:

(11) Es spart Schreibarbeit (und verbindert Rechenfehler), wenn wir uns vor Augen halten, dass wir den numerischen Wert der Massen des Meteoriten außer Betracht lassen können; die Masse ist ja invariant (Tipler / Llewelin).

Die Eigenschaft der Modalpartikeln besteht darin, dass sie immer die Sprechereinstellung kodieren. Auch wenn die Einstellung einer 3. Person wiedergegeben wird, wird die Position des Sprechers ausgedrückt, vgl. (12):

(12) Später im Text schreibt er dann das er ja sowieso net pennen könnte und ziemlich matschig ist!! (DECOW 16A)

- die Partikel ja kodiert sowohl die Sprechereinstellung als auch die Einstellung der 3. Person.

(13) Ich habe meine Mutter jahrelang nach dem Rezept gefragt und erhielt meist die Auskunft, das sei "ganz einfach, Du machst einen Hefeteig, kochst Grießbrei und streichst Schmandsoße drüber. Und dann Obst, das weißt Du ja"(DECOW 16A)

- die Modalpartikel ja wird im Redemodus gebraucht und kodiert die Sprechereinstellung. 
In illokutiv unselbständigen Nebensätzen werden Modalpartikeln nicht verwendet, was schon von Thurmair (1989), Coniglio (2011) und Averina (2015) gezeigt worden ist, vgl.:

(14a) Dann fiel ibr wieder ein, dass sie ja fliegen konnte (DECOW 16A).

$\left(14 \mathrm{~b}^{*}\right)$ Sie bedauerte, dass sie ja fliegen konnte.

Modalpartikeln betrachte ich als mediale Deiktika, da sie in einem narrativen Modus mit dem auktorialen Sprecher möglich sind.

\subsubsection{Modalpartikeln im Russischen}

Zum Ausdruck des Zweifels werden solche Partikeln wie mol, deskat', jakoby verwendet. Neben dem Zweifel wird darauf hingewiesen, dass die Aussage einem anderen gehört - die evidentielle Semantik wird mitkodiert. Zum Ausdruck der Epistemizität werden die Partikeln vrjad li, vrode kak, vrode kak by verwendet - sie drücken Zweifel aus. Für die Verstärkung der Aussage und für die Kodierung der Sicherheit des Sprechers dienen die Partikeln ved’und $\check{z} e$, vgl.:

(15) bylo nelegko, no ved'e to kak raz to, čto nužno (NKRJ).

,Es war nicht leicht, aber das ist ja genau das, was man braucht

(16) Tem ne menee èto vrjad li proizojdët (NKRJ).

,Nichtdestotrotz passiert das kaum

(17) Gusinye Lapki razdumyval, stoit li obsledovat' ostrov, na kotorom vrjad li pomestitsja slon [...] (NKRJ).

,Gusinye Lapki hat sich Gedanken darüber gemacht, ob es sich lohnt, die Insel zu untersuchen, auf der sich ein Elefant kaum platziert werden könntec

(18) Vrode kab by „živ, živ!” kričit kulik Kronšnep (NKRJ).

,Der Schlammläufer Kronschnep schnttert, dass er „lebendig!" zu sein scheint

(19) My že dogovorilis’: vse čëtnye tvoi, a nečëtnye moi! (NKRJ).

,Wir haben uns ja verabredet: Alle geraden gehören dir, alle ungeraden gehören mir ${ }^{`}$

Die genannten Partikeln sind für den Redemodus typisch (15), (16), (19), sie sind auch im Textmodus (17), (18) und in illokutiv selbständigen Nebensätzen möglich, in illokutiv unselbständige Nebensätze können sie nicht eingebettet werden, vgl.:

(20a) A do ètogo-to čitali byliny, čto vrode kak Muromec s detstva takim silnym byl (NKRJ). ,Und vorhin hat man Heldengedichte gelesen, dass Muromec vermutlich von Kindheit an so stark war'

(20b) On sožalel, čto (*vrode kak, *vrjad li, *vrode kak by) Moromec s detstva takim silnym byl. ,Er bedauerte, dass ( $\left.{ }^{*} M P\right)$ Muromec vermutlich von Kindheit an so stark war' 


\subsubsection{Deutsche Modalverben in sekundärer Bedeutung}

Modalverben in sekundärer (epistemischer) Bedeutung können in illokutiv selbständigen Nebensätzen sowie im narrativen Textmodus mit dem auktorialen Sprecher verwendet werden, vgl.:

(21a) „Das muss ein Irrtum sein“, sagten die beiden Kollegen (DECOW 16A).

(21b) Zwar warnte ein Mitglied vor dem Risiko, dass der neue Vorstand in zwei Jahren sagen müsse, er könne es nicht stemmen (DECOW 16A).

(21c) Ein Mitglied bedauerte, dass der neue Vorstand in zwei Jahren sagen ( ${ }^{*}$ müsse) muss, er kann es nicht stemmen.

In (21a) kodiert müssen die Semantik der Vermutung, das ist ein Redemodus. Im nächsten Satz (21b) wird müssen in epistemischer Lesart verwendet, der Satz ist illokutiv selbständig. In (21c) ist die epistemische Lesart von müssen wegen des faktiven Prädikats im Matrixsatz nicht akzeptabel. Auf die Tatsache, dass Modalverben in epistemischer Lesart in illokutiv unselbständigen Nebensätzen nicht verwendet werden können, hat AвRAHAM (2013: 35) hingewiesen. Modalverben in epistemischer Lesart können sowohl die Einstellung des auktorialen Sprechers als auch die Einstellung einer der Figuren kodieren:

(22) Sie musste irgend etwas davon fühlen, denn sie spannte ihre Augenbrauen hoch, wie wenn man jemand Lästigen wegweisen will... (S. Zweig, Der Amokläufer)

- die Vermutung wird von dem Sprecher geäußert;

(23) [...] das reizte mich schon einigermaßen, und als dann noch die deutsche Dame diese Lektion mit dem lehrhaften Senf bestrich, es gäbe einerseits wirkliche Frauen und anderseits „Dirnennaturen“, deren ihrer Ansicht nach Frau Henriette eine gewesen sein musste, da riss mir die Geduld vollends, ich wurde meinerseits aggressiv (S. Zweig, Vierundzwanzig Stunden aus dem Leben einer Frau)

- die Vermutung wird von einer der Figuren geäußert, in deren Rolle eine Dame auftritt (die deutsche Dame).

Modalverben in sekundärer Bedeutung zähle ich zu medialen Deiktika: Sie können im Redemodus, in einem narrativen Text mit dem auktorialen Sprecher sowie in illokutiv selbständigen Nebensätzen verwendet werden.

\subsubsection{Russische Modalverben in sekundärer Bedeutung}

Modalverben des Russischen moč, hotet', umet' und byt'dolžnym dienen in der Regel zum Ausdruck der voluntativen, aletischen und deontischen Modalität. Das Modalverb byt' dolžnym kann in zwei Bedeutungen benutzt werden: (1) Zum Ausdruck der Notwendigkeit, wie im Beispiel (24), und (2) für die Kodierung der Vermutung, wobei ein hoher Sicherheitsgrad ausgedrückt wird (25a), vgl.:

(24) Kuda ty sobralsja ehat'? Ty dolžen byt'zdes'(NKRJ). ,Wo willst du hin? Du musst hier sein 
(25)a. Priskakavšij eger' uverjaet, čto Abdallah brosilsja v rov i dolžen byt' zdes' nedaleko (NKRJ). ,Der angerannte Jäger versichert, dass Abdallah in den Graben hinstürzte und irgendwo hier sein muss

Zum Ausdruck der Epistemizität kann das Verb možet byt' verwendet werden. Es hat den Status eines Einführungswortes, vgl.:

(26) Možet byt', aktëry sozdajut eto pritjaženie (NKRJ).

,Vielleicht schaffen die Schauspieler diese Anziehung selbst

Bei der Verwendung des Prädikativs dolžen in einem illokutiv unselbständigen Nebensatz wird nicht die Epistemizität, sondern die Semantik der aletischen Modalität kodiert:

(25)b. On ošibaetsja, čto Abdallah brosilsja v rov i dolžen byt'zdes' nedaleko. ,Der angerannte Jäger versichert, dass Abdallah in den Graben hinstürzte und irgendwo hier sein muss' (= es besteht eine potentielle Möglichkeit, dass er hier ist).

Das System der Modalverben im Russischen ist mit dem System der Modalverben im Deutschen nicht identisch: Nur zwei Modalverben können manchmal in epistemischer Lesart auftreten; häufig ist es nur in Kombination mit dem Verb byt' / sein möglich; in der Regel haben sie den Status eines Einführungswortes.

\subsubsection{Modaladverbien im Russischen}

Im Russischen gibt es viele Modaladverbien, die Epistemizität kodieren können, z.B. vozmožno, verojatno, vidimo, kažetsja, po usej vidimost, navernoe, navernjaka, dejstvitel'no, samo soboj, konečno usw. Diese Wörter können sowohl im Redemodus als auch im Textmodus verwendet werden, vgl.:

(27) Po vsej vidimosti, svoego šansa on ne upustit (NKRJ). ,Anscheinend verliert er seine Chance nicht

(28) Eto vsë iz-za kompjutera, navernoe (NKRJ). ,Das ist vielleicht wegen des Computers

Die genannten Modaladverbien können nur in illokutiv selbständigen Nebensätzen verwendet werden (29a) - ihr Gebrauch in illokutiv unselbständigen Nebensätzen ist nicht akzeptabel (29b), vgl.:

(29a) Sejčas on chotel skazat', čto, vidimo (+verojatno, +navernoe, +dejstvitel'no, +samo soboj), ego skem-to pereputali (NKRJ).

,Jetzt wollte er sagen, dass man ihn anscheinend mit jemandem verwechselt hat

(29b) On sožalel, čto ego ( ${ }^{*}$ verojatno, * navernoe, *dejstvitel'no, * samo soboj) s kem-to pereputali. ,Er bedauerte, dass man ihn ( ${ }^{*}$ Modaladverb) mit jemandem verwechselt hat'. 


\subsection{Entfernte Egozentrika: Modaladverbien im Deutschen}

Zu den entfernten Egozentrika würde ich Modaladverbien im Deutschen zählen, da sie fast in allen Modi verwendet werden können: im Redemodus, im Textmodus mit dem auktorialen Sprecher sowie in illokutiv selbständigen Nebensätzen, vgl.:

(30) ich schreibe Ihnen als Mitarbeiter des YUNA Service Teams und bedauere sehr, dass Sie offensichtlich ein Problem mit Ihrer YUNA Card hatten (DECOW 16A).

(31) Wir schreiben hastig, machen Fehler und benutzen bunte Bilder dort, wo vielleicht Worte besser gewesen wären (DECOW 16A).

(32) Sie ließe Ihren Namen da, wo er wabrscheinlich früh oder spät noch einmal zu lesen sein wird (DECOW 16A).

(33) So sollte es auch heute sein, und Grenouilles Mutter, die noch eine junge Frau war, gerade Mitte zwanzig, die noch ganz hübsch aussah und noch fast alle Zühne im Munde hatte und auf dem Kopf noch etwas Haar und außer der Gicht und der Syphilis und einer leichten Schwindsucht keine ernsthafte Krankheit; die noch hoffte, lange zu leben, vielleicht fünf oder zehn Jahre lang, und vielleicht sogar einmal zu heiraten und wirkliche Kinder zu bekommen als ehrenwerte Frau eines verwitweten Handwerkers oder so (P. Süskind, Das Parfum).

Am Beispiel der angeführten Belege können wir die Verwendung von Modaladverbien in illokutiv unselbständigen Nebensätzen sowie im Textmodus mit dem auktorialen Sprecher beobachten. So ist z.B. der Objektsatz in (30) illokutiv unselbständig, in (31) weist das Korrelat dort auf die illokutive Selbständigkeit des Lokalsatzes, in (32) erfüllt diese Funktion das Korrelat da. In (33) wird das Modaladverb vielleicht im narrativen Textmodus mit dem auktorialen Sprecher verwendet, indem die Einstellung der dritten Person - der Mutter von Grenuille - kodiert wird.

Es ergibt sich, dass es Unterschiede zwischen den Modaladverbien im Deutschen und im Russischen gibt: Modaladverbien im Russischen können nicht in illokutiv unselbständigen Nebensätzen verwendet werden, während das für deutsche Modaladverbien möglich ist.

\section{Fazit}

Im vorliegenden Beitrag habe ich die Korrelation solcher Entitäten wie Deixis und Egozentrizität behandelt und Epistemizitätsmarker des Deutschen und des Russischen nach ihrer Entfernung von der Origo differenziert. Die durchgeführte Analyse lässt folgende Schlussfolgerungen machen:

1. Intonatorische Mittel der Kodierung von Epistemizität gehören zu nahen Egozentrika, da sie nur im Redemodus möglich sind. Sie sind auch in illokutiv selbständigen Sätzen akzeptabel. Für das Deutsche sind das der Verumfokus und betonte Partikeln, denen im Russischen die Aussagen mit dem verifikativen Rhema entsprechen.

2. Modalpartikeln im Deutschen und im Russischen, Modaladverbien im Russischen sowie deutsche Modalverben in epistemischer Lesart kann man zur Gruppe der medialen 
Egozentrika zählen: Sie sind in illokutiv selbständigen Nebensätzen, im Redemodus sowie im Textmodus mit dem auktorialen Sprecher möglich. Ihre Eigenschaft besteht darin, die Einstellung des Sprechers oder der 3. Person wiederzugeben. Das System der Modalverben im Russischen ist mit dem System der Modalverben im Deutschen nicht identisch - von russischen Modalverben wird vorwiegend aletische oder deontische Modalität kodiert.

3. Deutsche Modaladverbien gehören zur Gruppe der sekundären Egozentrika, da sie in allen Interpretationsmodi ohne Einschränkungen verwendet werden können, was sie von Modaladverbien im Russischen unterscheidet.

\section{Quellen}

DECOW 16 A. URL: www.webcorpora.org [15.07.2018-15.08.2018].

NKRJ (Das Nationalkorpus der russischen Sprache). URL: http://www.ruscorpora.ru/ [17.07.2018-18.08.2018].

Süskind, Patrick (1994): Das Parfum. Die Geschichte eines Mörders. Zürich: Diogenes.

Tipler, A.P. / Llewelin R.A. (2010): Moderne Physik. München: Oldenbourg Wissenschaftsverlag. Zweig, Stefan (1989): Der Amokläufer. Frankfurt/M.: Fischer.

Zweig, Stefan (2011): Vierundzwanzig Stunden aus dem Leben einer Frau. Frankfurt/M.: Fischer.

\section{Bibliographie}

Aвraham, Werner (2012): Sprecherdeixis und Merkmalsdistributionsdifferential deutscher Modalitätselemente. In: Deutsche Sprache 40, 200-231.

Abraham, Werner (2013): Zur grammatischen Grundlegung von Modalität - semantisch-syntaktische Affinitäten zu nominaler Referenz, Aspekt und Quantifikation. In: AвRAHAM, Werner / LeIss, Elisabeth (Hg.): Funktionen von Modalität. Berlin: De Gruyter, 25-76.

Abraham, Werner (2017): Modal particles and Verum focus. New corollaries. In: Fedriani, Chiara / SAnsò, Andrea (Hg.): Pragmatic Markers, Discourse Markers and Modal Particles. Amsterdam: John Benjamins, 171-202.

AdAmec, Přemysl (1966): Porjadok slov v sovremennom russkom jazyke. Praha: Academia Nacladatelstvi československé academie věd.

ApresjAn, Jurij D. (1995): Dejksis v leksike i grammatike i naivnaja model' mira. In: Izbrannye trudy. Moskva: Jazyki russkoj kultury, 629-650.

Averina, Anna (2015): Partikeln im komplexen Satz. Mechanismen der Lizenzierung von Modalpartikeln in Nebensätzen und Faktoren ibrer Verwendung in komplexen Sätzen. Am Beispiel der Modalpartikeln „ja“, „doch“ und „denn“im Deutschen und „ved”“, „že“ und „vot“im Russischen. Frankfurt/M.: Peter Lang.

BLÜHDORN, Hardarik (2012): Faktizität, Wahrheit, Erwünschtheit: Negation, Negationsfokus und „Verum“-Fokus im Deutschen. In: LohnsteIn, Horst / BlüHdorn, Hardarik (Hg.): Wahrheit, Fokus, Negation. Hamburg: Buske, 137-170. 
BüHLER, Karl (1978): Sprachtheorie. Die Darstellungsfunktion der Sprache. Ulm: Ullstein Buch.

Coniglio, Marco (2011): Die Syntax der deutschen Modalpartikeln. Ihre Distribution und Lizenzierung in Haupt-und Nebensätzen. Berlin: Akademieverlag.

Diewald, Gabriele (1991): Deixis und Textsorten im Deutschen. Tübingen: Niemeyer.

DIEWALD, Gabriele (2013): Modus und Modalverben Kategorisierungsoptionen im grammatischen Kernbereich der Modalität. In: AbRAh AM, Werner / Leiss, Elisabeth (Hg.): Funktionen der Modalität. Berlin: De Gruyter, 77-110.

EgG, Marcus / Zimmermann, Malte (2011): Stressed Out! Accented discourse particles: the case of doch. In: Aguilar, Ana / Chernilovskaya, Anna / Nouwend, Rick (Hg.): Proceedings of Sinn und Bedeutung 16. University of Utrecht, 225-238.

Fillmore, Charles (1981): Pragmatics and the Description of Discource. In: Cole, Peter (ed.): Radical Pragmatics. New York: Academia Press, 143-166.

Höhle, Tilman N. (1988): Verum-Fokus. In: Sprache und Pragmatik 5. Arbeitsberichte. Lund, 1-8.

HöHLE, Tilman N. (1992): Über Verum-Fokus im Deutschen. In: JAcoBs, Joachim (Hg.): Informationsstruktur und Grammatik (Linguistische Berichte. Sonderheft 4), 112-141.

JaNKo, Tatjana E. (2008): Intonacionnye strategii russkoj reči v sopostavitel'nom aspekte. Moskva: Jazyki slavjanskich kultur.

LEISs, Elisabeth (2011): Lexikalische versus grammatische Epistemizität und Evidentialität: Plädoyer für eine klare Trennung von Lexikon und Grammatik. In: Diewald, Gabriele / Smirnova, Elena (Hg.): Modalität und Evidentialität (Fokus 37). Trier: Wissenschaftlicher Verlag, 149-169.

Meibauer, Jörg (1994): Modaler Kontrast und konzeptuelle Verschiebung. Studien zur Syntax und Semantik deutscher Modalpartikeln. Tübingen: Niemeyer.

Padučeva, Elena V. (2010): Semantičeskie issledovanija. Semantika vremeni i vida v russkom jazyke. Semantika narrativa. Moskva: Jazyki slavjanskoj kultury.

Stommel, Hildegard (2012): Verum-Fokus als Kontrast-Fokus. In: LoHnsteIn, Horst / BLÜHdorn, Hardarik (Hg.): Wahrheit, Fokus, Negation. Hamburg: Buske, 15-29.

Thurmair, Maria (1989): Modalpartikeln und ibre Kombinationen. Tübingen: Niemeyer. 